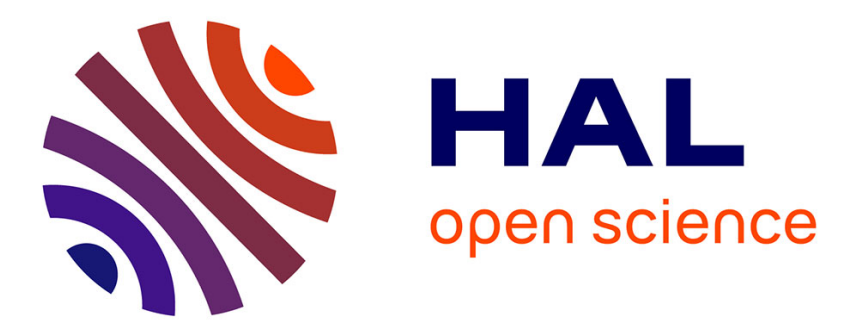

\title{
SIR2 Expression Noise Can Generate Heterogeneity in Viability but Does Not Affect Cell-to-Cell Epigenetic Silencing of Subtelomeric URA3 in Yeast
}

\author{
Jian Liu, Laureline Mosser, Catherine Botanch, Jean Marie François, \\ Jean-Pascal Capp
}

\section{To cite this version:}

Jian Liu, Laureline Mosser, Catherine Botanch, Jean Marie François, Jean-Pascal Capp. SIR2 Expression Noise Can Generate Heterogeneity in Viability but Does Not Affect Cell-to-Cell Epigenetic Silencing of Subtelomeric URA3 in Yeast. G3, 2020, 10 (9), pp.3435-3443. 10.1534/g3.120.401589 . hal-02968099

\section{HAL Id: hal-02968099 \\ https: / hal.inrae.fr/hal-02968099}

Submitted on 15 Oct 2020

HAL is a multi-disciplinary open access archive for the deposit and dissemination of scientific research documents, whether they are published or not. The documents may come from teaching and research institutions in France or abroad, or from public or private research centers.
L'archive ouverte pluridisciplinaire HAL, est destinée au dépôt et à la diffusion de documents scientifiques de niveau recherche, publiés ou non, émanant des établissements d'enseignement et de recherche français ou étrangers, des laboratoires publics ou privés. 


\title{
SIR2 Expression Noise Can Generate Heterogeneity in Viability but Does Not Affect Cell-to-Cell Epigenetic Silencing of Subtelomeric URA3 in Yeast
}

\author{
Jian Liu, Laureline Mosser, Catherine Botanch, Jean-Marie François, and Jean-Pascal Capp ${ }^{1}$ \\ Toulouse Biotechnology Institute, University of Toulouse, INSA, CNRS, INRAE, Toulouse, France \\ ORCID IDs: 0000-0002-4914-7025 (J.L.); 0000-0002-6470-079X (J.-P.C.)
}

\begin{abstract}
Chromatin structure clearly modulates gene expression noise, but the reverse influence has never been investigated, namely how the cell-to-cell expression heterogeneity of chromatin modifiers may generate variable rates of epigenetic modification. Sir2 is a well-characterized histone deacetylase of the Sirtuin family. It strongly influences chromatin silencing, especially at telomeres, subtelomeres and rDNA. This ability to influence epigenetic landscapes makes it a good model to study the largely unexplored interplay between gene expression noise and other epigenetic processes leading to phenotypic diversification. Here, we addressed this question by investigating whether noise in the expression of SIR2 was associated with cell-to-cell heterogeneity in the frequency of epigenetic silencing at subtelomeres in Saccharomyces cerevisiae. Using cell sorting to isolate subpopulations with various expression levels, we found that heterogeneity in the cellular concentration of Sir2 does not lead to heterogeneity in the epigenetic silencing of subtelomeric URA3 between these subpopulations. We also noticed that SIR2 expression noise can generate cell-to-cell variability in viability, with lower levels being associated with better viability. This work shows that SIR2 expression fluctuations are not sufficient to generate cell-to-cell heterogeneity in the epigenetic silencing of URA3 at subtelomeres in Saccharomyces cerevisiae but can strongly affect cellular viability.
\end{abstract}

\author{
KEYWORDS \\ Epigenetics \\ SIR2 \\ silencing \\ gene expression \\ noise \\ viability
}

Noise in gene expression is an important contributor to phenotypic diversification and can have important consequences in processes ranging from the developmental to the evolutionary level (Ackermann 2015; Raj and van Oudenaarden 2008). However, its relationships with other processes that produce phenotypic diversity by acting at the genetic or epigenetic level are only partly understood. The influence of genetic and epigenetic variation on noise is well characterized (Sanchez et al. 2013; Sanchez and Golding 2013) but the reverse relationship has seldom been explored.

Copyright ( 92020 Liu et al.

doi: https://doi.org/10.1534/g3.120.401589

Manuscript received June 30, 2020; accepted for publication July 23, 2020; published Early Online July 28, 2020.

This is an open-access article distributed under the terms of the Creative Commons Attribution 4.0 International License (http://creativecommons.org/ licenses/by/4.0/), which permits unrestricted use, distribution, and reproduction in any medium, provided the original work is properly cited.

Supplemental material available at figshare: https://doi.org/10.25387/ g3.12724955.

${ }^{1}$ Corresponding author: TBI, University of Toulouse, INSA, CNRS, INRAE,

Toulouse, France. E-mail: capp@insa-toulouse.fr
The level of expression noise is particularly influenced by promoter architecture. Genes with TATA-box containing promoters show higher noise levels than do other genes in yeast and mammals (Newman et al. 2006; Zhang et al. 2009; Zoller et al. 2015). Mutating the TATA box clearly decreases promoter-mediated noise (Blake et al. 2006; Fraser et al. 2004; Hornung et al. 2012). The strength, number and position of transcription factor binding sites also affect noise: promoters with more TF binding sites have higher expression noise for instance (Sharon et al. 2014; To and Maheshri 2010). Finally, another factor that has remarkable effects on expression noise is the positioning of nucleosomes on promoters. A promoter with nucleosome binding sites has silenced or open alternative states. The transient opening and reclosing of these promoters lead to transcriptional bursting events of variable duration and frequency, generating cell-to-cell variability in gene expression (Sanchez and Golding 2013). Promoters with polynucleosome-disfavoring sequences thus have less noise because higher transcription burst frequencies lead to lower cell-to-cell heterogeneity (Sharon et al. 2014).

These data suggest that epigenetic changes play an important role. Moreover, results from single-cell nucleosome mapping on the same promoter revealed significant cell-to-cell variation in nucleosome 
positions during induction of the $\mathrm{PHO} 5$ gene in yeast (Small et al. 2014). Nucleosome positioning complexity may thus contribute to the flexibility and heterogeneity of gene expression, independently of the promoter sequence. A qualitative model for nucleosome positioning in Saccharomyces cerevisiae has shown that nucleosome architecture is indicative of the amount of transcriptional noise in TATA-box-containing promoters (Zaugg and Luscombe 2012). Also, increased eviction or sliding of promoter nucleosomes makes the promoter more accessible to transcriptional machinery in the initiation of transcription and thus probably reduces expression noise during induction (Rawal et al. 2018). Other results highlight the role of the chromatin environment in the modulation of expression noise: for instance, changing the location of a gene changes its level of noise in S. cerevisiae (Becskei et al. 2005), Candida albicans (Anderson et al. 2014) and chicken cells (Viñuelas et al. 2013). Mean expression and noise are uncorrelated across genomic locations in mammalian cells and the chromatin environment clearly influences the level of noise. More repressed chromatin is associated with higher expression noise (Dey et al. 2015). Mutations in chromatin remodelers also affect this phenomenon in yeast (Fraser et al. 2004; Weinberger et al. 2012). Finally, heterochromatin formation has been suggested to be locus autonomous and to be established stochastically in $S$. cerevisiae (Xu et al. 2006). Similarly, triggered spreading of heterochromatin is stochastic, multimodal, and fluctuates dynamically over time in fission yeast (Greenstein et al. 2018; Obersriebnig et al. 2016). However, another study has found that fluctuations in heterochromatic silencing are not locus autonomous (Mano et al. 2013), revealing a lack of consensus on this point. In any case, the lack of stability correlates with high histone turnover, showing that cell-tocell heterogeneity in the silencing status of precise loci is mainly controlled by the dynamics of the chromatin environment (Greenstein et al. 2018; Obersriebnig et al. 2016).

In contrast, much less is known about how gene expression noise influences the rates of genetic and epigenetic modifications. Gene expression noise directly influences genetic variability in Escherichia coli because response to DNA alkylation damage and the subsequent mutagenesis depend on the stochastic expression of Ada (Uphoff et al. 2016); and in S. cerevisiae, where cell-to-cell heterogeneity in the homologous recombination rate stems from noise in the expression of genes such as RAD52 and RAD27 (Liu et al. 2019). However, the influence of noise in the expression of epigenetic regulators on chromatin modification activity has never been explored.

Sir2 is a well-known histone modifier of the Sirtuin family, largely characterized in $S$. cerevisiae for its ability to perform epigenetic silencing at silent mating-type loci (HML/HMR), telomeres, subtelomeres, and rDNA through its NAD+ dependent histone deacetylase activity (Grunstein and Gasser 2013). This well-conserved protein from eubacteria and archaea to man associates with Sir3 and Sir4 in the SIR complex to perform silencing in S. cerevisiae by acting on the N-terminal tails of histones H3 and H4 (Grunstein and Gasser 2013; Newcomb and Bedalov 2009). Interestingly, it has been shown to influence the expression noise level of subtelomeric TLO genes in $C$. albicans by modulating the shift between silent and active chromatin states (Anderson et al. 2014). In S. cerevisiae, epigenetic silencing processes are commonly studied using telomere silencing assays on strains in which either URA3 or ADE2 is inserted in subtelomeres in a previous $\Delta$ ura 3 or $\Delta$ ade2 background (Grunstein and Gasser 2013). Typically, these assays are used to identify inhibitors of chromatin modifying enzymes (Newcomb and Bedalov 2009), notably for anticancer drug discovery (Simon and Bedalov 2004). Other loci are commonly used to test silencing. For instance, cellular levels of
Sir2 regulate the extent of gene silencing observed at the rDNA (Smith et al. 1998).

SIR2 thus provides a good model to test the influence of expression noise on epigenetic variability. We chose to investigate the epigenetic silencing of a URA3 cassette inserted in subtelomeres as a function of cellular levels of Sir2. We show that variable Sir2 expression does not generate heterogeneity in the epigenetic silencing of subtelomeric URA3 between cells. Nevertheless, SIR2 expression noise creates heterogeneity in viability, which decreases progressively with increases in Sir2 levels across the whole expression distribution. Thus, while SIR2 expression fluctuations are not sufficient to produce cell-to-cell heterogeneity in the epigenetic silencing of subtelomeric $U R A 3$, they can generate cell-to-cell heterogeneity in viability in $S$. cerevisiae.

\section{MATERIAL AND METHODS}

\section{Yeast constructs and growth conditions}

All the primers used in this article are listed in Table S1. The strain UCC2210 (Mat $\alpha$; ade2 $\Delta::$ hisG; his $3 \Delta 200$; leu2 $\Delta 0$; lys $2 \Delta 0$; met15 $\Delta 0$; $\operatorname{trp} 1 \Delta 63$; ura3 $\Delta 0$; adh4::URA3-TEL (VII-L); ppr1::HIS3) was used to measure URA3 silencing activity at subtelomeres (frequency of $U R A 3$ expression). To create the $\Delta$ sir 2 strain, a PCR fragment containing the LYS2 gene and homologous to SIR2 was amplified from the genomic DNA of the S288c strain with primers D1 and D2, and transformed into UCC2210. The construct was verified by PCR with primers V1 and V2. To create the C-terminal fusion strain Sir2-tdTomato, a PCR fragment containing tdTomato-KanR and homologies of the end of SIR2 was amplified with primers $\mathrm{C} 1$ and $\mathrm{C} 2$ from the plasmid pfa6atdTomato-KanR (made in our laboratory), and transformed into UCC2210. The constructs were verified by PCR with primers V3 and V4. The N-terminal fusion of SIR2 was created using a two-step strategy, taking advantage of the selection and anti-selection of the LYS2 gene. First, a PCR fragment containing the LYS2 gene and homologous to the beginning of SIR2 was amplified from the genomic DNA of the S288c strain with primers N1 and N2, and transformed into the strain UC2210. The constructs were verified by PCR with primers V5 and V2. Second, a synthesized fragment (Eurofins, Figure S1) containing tdTomato and homologous to the beginning of SIR2 was transformed to the previous strain to create the $\mathrm{N}$-terminal fusion (selection on alpha-aminoadipate). The construct was verified by PCR with primers V5 and V4.

To integrate an additional copy of SIR2 in UCC2210, the LEU2 gene was first reintroduced into the genome after amplifying LEU2 from the S288C genome using primers V6 and V7 and integrating it into UCC2210. A PCR fragment containing the SIR2 gene was then amplified from the genomic DNA of the S288c strain with primers V8 and V9 respectively containing the SalI et NotI restriction sites. SIR2 was then inserted in the yeast chromosomal integration vector pJRL2 (Addgene) previously modified in our laboratory to replace the selection cassette his-URA3-kanR-his by kanMX4 only (Liu et al. 2015) and digested using SalI and NotI. After ligation, the resulting plasmid was linearized by AscI digestion (restriction site between the regions homologous to the LEU2 locus) and transformed in UCC2210 using the lithium acetate method. Recombinants were selected on YPD + G418 agar plates and insertion was verified by PCR with primers V10 and V11. The strain was called UCC2210+SIR2inLEU2.

For creating the tdTomato-Tsl1 strain, the tdTomato sequence was amplified by PCR from the plasmid pfa6a-tdTomato-KanR with primers overlapping $50 \mathrm{bp}$ downstream and upstream the 
TSL1 start codon (primers T1 and T2, respectively). tdTomato was integrated using a CRISPR-Cas9 strategy. A plasmid carrying the gene coding for the Cas9 enzyme, LEU2 for auxotrophic selection (derived from pML107, Addgene) and containing an appropriate gRNA obtained with primers T3 and T4 and inserted in the plasmid thanks to the SapI cloning site, was transformed in the BY4741 strain (MATa; his $3 \Delta 1$; leu $2 \Delta 0$; met $15 \Delta 0$; ura $\Delta \Delta 0$ ) together with tdTomato amplified with primers T1 and T2. PAM cleavage site was determined with the CRISPR Direct webtool for gRNA sequence design (https://crispr.dbcls.jp/).

All the strains were grown in liquid YNB medium $(20 \mathrm{~g} / \mathrm{L}$ glucose (Sigma), $1.71 \mathrm{~g} / \mathrm{L}$ yeast nitrogen base without amino acids or nitrogen (Euromedex) and $5 \mathrm{~g} / \mathrm{L}$ ammonium sulfate (Sigma)) supplemented with dropout solution corresponding to the selection marker at $30^{\circ}$ with vigorous shaking (200 rpm).

The YPD plates used for viability measurements contained $20 \mathrm{~g} / \mathrm{L}$ glucose, $20 \mathrm{~g} / \mathrm{L}$ agar (Euromedex), $10 \mathrm{~g} / \mathrm{L}$ peptone (Euromedex) and $10 \mathrm{~g} / \mathrm{L}$ yeast extract (Euromedex). The SCD-ura plates used for URA3 expression measurements contained $20 \mathrm{~g} / \mathrm{L}$ glucose, $20 \mathrm{~g} / \mathrm{L}$ agar, 1.71 $\mathrm{g} / \mathrm{L}$ yeast nitrogen base, $5 \mathrm{~g} / \mathrm{L}$ ammonium sulfate, and $0.77 \mathrm{~g} / \mathrm{L} \mathrm{CSM}$ URA-' (Euromedex).

\section{Western blotting}

Western blotting analyses were performed using standard protocols. Total cellular protein $(100 \mu \mathrm{g})$ was separated by electrophoresis in a $10 \%$ SDS-PAGE gel and transferred to nitrocellulose membrane. Sir2 was detected using a goat polyclonal antibody (yN-19, Santa Cruz Biotechnology) followed by incubation with horseradish peroxidase (HRP)-conjugated anti-goat IgG (Abcam). Equal loading was verified and normalization was performed on two replicates using stain-free imaging technology and the Image Lab software (Bio-Rad).

\section{Fluorescence activated cell sorting}

An overnight culture was diluted 20 times and grown to exponential phase $(6 \mathrm{~h})$ to measure the fluorescence profile of the UCC2210, Sir2tdTomato, tdTomato-Sir2, BY4741, Rad27-tdTomato and Rad52tdTomato strains by MACSQuant VYB with the MACSQuantify Software (Miltenyi Biotec). A total of $10^{5}$ cells was analyzed for each strain, and the fsc files were exported and analyzed using the software $\mathrm{R}$ (v3.2) with the Bioconductor packages (v3.0). A norm2Filter filter was applied on FSC-A/SSC-A to select homogeneous cells in terms of size, shape, and cellular complexity. tdTomato fluorescence (Channel Y2-A) was log-transformed. All the figures were prepared using the transformed data. Each measurement was repeated three times.

The cell sorting experiments were performed on a MoFlo Astrios EQ cell sorter and analyzed with the Summit v6.3 software (Beckman Coulter). Cells in stationary phase were diluted 100 times and grown at $30^{\circ}$ with vigorous shaking $(200 \mathrm{rpm})$ for $16 \mathrm{~h}$ prior to cell sorting (final $\mathrm{OD} \approx 2$ ). Cultures were spun down at $3000 \mathrm{~g}$ for five minutes at $4^{\circ}$. Growth media were removed and the cells were re-suspended in ice cold PBS. The SmartSampler and CyClone tube holders were kept at $4^{\circ}$ during cell sorting. Cell sorting was carried out with a $70 \mu \mathrm{m}$ nozzle at an operating pressure of 60 psi. The sorting speed was kept at around 30000 events per second. The purity mode was chosen for sorting along with a single-drop droplet envelope. Single cells of similar size and granularity were first selected based on the FSC-Area vs. SSC-Area (488 nm laser) plot and the FSC-Height vs. FSC-Area (488 nm laser) plot. Then, based on the tdTomato fluorescence histogram (560 nm laser, 614/20 filter), single cells were simultaneously sorted either into the $2 \%$ most and least fluorescent (Figure 2A), or into five subpopulations defined in terms of their fluorescence as follows: $0-20 \%, 20-40 \%, 40-60 \%, 60-80 \%$ and $80-100 \%$ (only for viability analysis) (Figure $4 \mathrm{~B}$ ).

To analyze the dynamics of recovery of the initial gene expression profile from the sorted extreme subpopulations, the UCC2210:tdTomato-Sir2 strain was grown overnight at $30^{\circ}$ in YPD medium and diluted 10 times in the morning. After $3 \mathrm{~h}, 6.10^{5}$ cells from the bottom cells and the top $2 \%$ were sorted simultaneously with MoFlo Astrios EQ (Beckman Coulter). The unimodality of the sorted cells was verified and they were then grown at $30^{\circ}$ in YPD medium. The dynamics of expression recovery was monitored with a MACSQuant VYB flow cytometer (Miltenyi Biotec) for $6 \mathrm{~h}$ or $24 \mathrm{~h}$.

\section{Measurement of survival frequency on SCD-ura plates and of viability on YPD plates}

To measure the frequency of survival on SCD-ura plates in whole populations, $100 \mu \mathrm{L}$ of 100 times diluted UCC2210, tdTomato-Sir2 and UCC2210+SIR2inLEU2 cultures (exponential phase at OD 1.5); and $100 \mu \mathrm{L}$ of 10000 times diluted $\Delta$ sir 2 and Sir2-tdTomato cultures (exponential phase at OD 1.5) were spread on SCD-ura plates. At the same time, $20 \mu \mathrm{L}$ of 10000 times diluted culture was spread on YPD plates for all the strains. The plates were kept in a $30^{\circ}$ incubator for 3 days and the number of clones was counted. As survival is most likely due to epigenetic URA3 activation, we estimated the frequency of URA3 expression for UCC2210, tdTomato-Sir2 and UCC2210+SIR2inLEU2 as follows:

$$
f=\frac{n_{-u r a} \times 20}{n_{Y P D} \times 10000}
$$

where $f$ denotes the frequency of URA3 expression, $n_{- \text {ura }}$ denotes the number of clones on the SCD-ura plates, and $n_{Y P D}$ denotes the number of clones on the YPD plates.

The frequency of URA3 expression for $\Delta$ sir2 and Sir2-tdTomato was calculated as follows:

$$
f=\frac{n_{-u r a} \times 20}{n_{Y P D} \times 100}
$$

To measure the frequency of URA3 expression in the sorted subpopulations with different expression levels, 10000 cells from each subpopulation were sorted and spread on SCD-ura plates, and 150 cells were then sorted and spread on YPD plates. The plates were kept in a $30^{\circ}$ incubator for 3 days and the number of clones was counted. The frequency of URA3 expression was calculated as follows:

$$
f=\frac{n_{-u r a} \times 150}{n_{Y P D} \times 10000}
$$

The viability was calculated as follows:

$$
v=\frac{n_{Y P D}}{n_{\text {plated }}}
$$

where $v$ denotes the viability, $n_{Y P D}$ denotes the number of clones on the YPD plates and $n_{\text {plated }}$ denotes the number of plated cells to estimate viability.

All the experiments were repeated at least three times and three technical replicates were done for each sample in each experiment (the number of clones given in each experiment for each sample is the mean of these three technical replicates). 


\section{Statistical analysis}

Results were mainly compared using two-tailed Student's $t$-tests on the data of three independent replicates. Results are presented as mean \pm SD and $p$ values as ${ }^{*} P<0.05$, ${ }^{* *} P<0.01$ and ${ }^{* * *} P<0.005$. The Pearson correlation coefficient between expression and viability was calculated using Microsoft Excel.

\section{Data availability}

Strains are available upon request. Figure S1 shows the sequence of the synthetized tdTomato gene with flanking homologies to SIR2. Figure S2 illustrates the growth of clones with subtelomeric URA3 either on SCD-ura plates or on 5-FOA plates. Figure S3 and S4 describe the recovery of the initial expression profile after cell sorting for both low and high Sir2-expressing cells, after $6 \mathrm{~h}$ and $24 \mathrm{~h}$ respectively. Figure S5 shows the viability of subpopulations of the tdTomato-Tsl1-tagged strain expressing extreme tdTomato levels similar than in the tdTomato-Sir2-tagged strain. Table S1 lists the primers used in this study. Table S2 contains the raw data of the silencing and viability analyses. Supplemental material available at figshare: https://doi.org/10.25387/g3.12724955.

\section{RESULTS}

\section{SIR2 expression noise does not generate cell-to-cell heterogeneity in the epigenetic silencing frequency of subtelomeric URA3}

As a fluorescent signal above the auto-fluorescence background was needed to efficiently sort Sir2-expressing cells, we chose tdTomato to tag Sir2 because it is one of the brightest fluorescent proteins available. We fused tdTomato either to the N-terminal or to the C-terminal domain of Sir2 to select the fused protein with the highest fluorescence and the best functionality. The fluorescence distribution of both fused proteins overlapped slightly with the auto-fluorescence background, and only slight differences in fluorescence were observed between the fusions, the $\mathrm{N}$-terminal fusion being the most fluorescent (Figure 1A).

We also tested the functionality of the fused proteins in subtelomeric silencing assays by using the reporter strain containing URA3 in the vicinity of a telomere (Figure $1 \mathrm{~B}$ ) where its transcription is modulated by epigenetic silencing (Grunstein and Gasser 2013). Silencing of URA3 allows growth in the presence of 5-fluoroorotic acid (5-FOA), because the Ura3 gene product converts 5-FOA to 5-fluorouracil, an inhibitor of DNA synthesis and disruptor of RNA processing (Lum et al. 2004; Giaever et al. 2004) that causes cell death. The frequency of URA3 repression can thus be scored accurately on plates that contain 5-FOA (Grunstein and Gasser 2013). On the contrary, if the strain lacks Ppr1, a strong activator of URA3, URA3 is globally repressed and is only expressed at low levels. In the pprl strain, only perturbed silencing can permit sufficient URA3 transcription for growth in a medium lacking uracil, but this occurs at low frequency and the whole population grows poorly in this medium (Newcomb and Bedalov 2009). This means that the frequency of URA3 expression can be scored on plates that lack uracil (Grunstein and Gasser 2013).

The strain used in this work (UCC2210) lacks Ppr1. We therefore selected URA3 expressing cells on uracil-deficient plates because only cells in which URA3 is not silenced grow efficiently enough to form countable colonies on this medium (Figure S2). In our experimental conditions, the frequency of URA3 expression in the UCC2210 strain with the URA3 telomeric reporter was about $8.10^{-3}$, but increased markedly when SIR2 was deleted, showing that acquisition of URA3 expression is of epigenetic origin (Figure 1C and Table S2). While the fluorescence levels of both tdTomato fused proteins were close

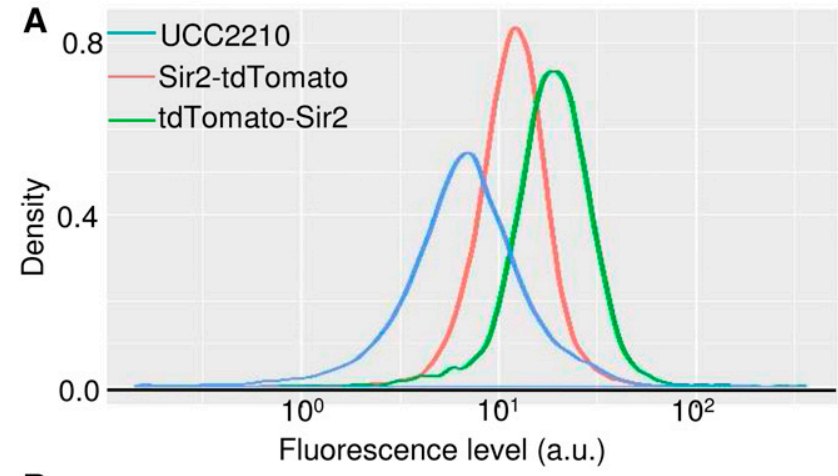

B

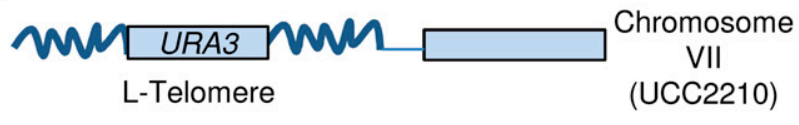

C

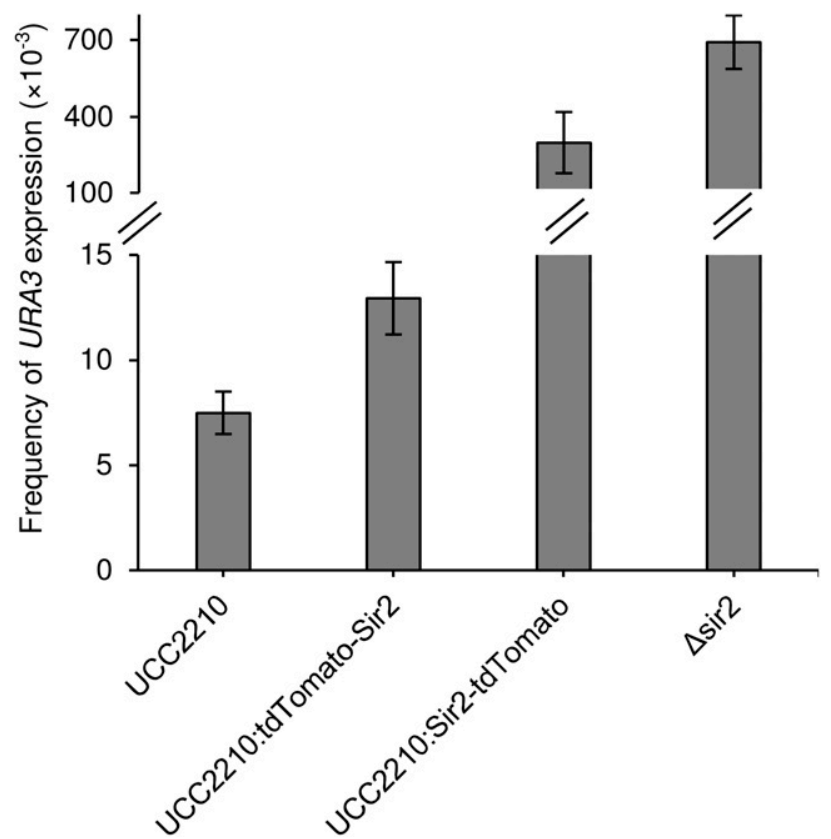

Figure 1 Fluorescence and subtelomeric URA3 silencing frequency of strains expressing Sir2 fused to tdTomato., A) Fluorescence profiles of the non-fluorescent control UCC2210 containing the subtelomeric URA3 silencing substrate, and of its derivatives with SIR2 fused to tdTomato at its original genomic locus either $\mathrm{N}$-terminally (tdTomatoSIR2) or C-terminally (SIR2-tdTomato). B) The subtelomeric URA3 silencing substrate consists of a URA3 gene inserted in the vicinity of a telomere where its transcription is modulated by epigenetic silencing. As the strain used lacks Ppr1, which is a strong activator of URA3, positive selection can be performed on uracil-deficient plates where only URA3-expressing cells can grow., C) Frequency of URA3 expression for the non-fluorescent control UCC2210, N-terminal tagged tdTomato-Sir2 strain, C-terminal tagged Sir2-tdTomato strain, and $\Delta$ sir2 strain. The results shown are the means and standard deviations of three independent experiments.

(Figure 1A), the frequency of URA3 expression was closer to that of the control strain for the N-terminal fusion, suggesting that it had better protein functionality than the C-terminal fusion (Figure 1C and Table S2). We therefore performed the sorting experiments with the N-terminal fused protein. 
Among the cells with heterogeneous expression levels of SIR2 at the single-cell level, we isolated the subpopulations (2\%) with the highest and lowest fluorescence intensities (Figure 2A). The frequency of URA3 expression was then evaluated using the URA3 telomeric reporter by measuring the frequency of cells growing on SCD-ura plates normalized by the viability (Figure 2A). No difference between the Sir2-low and Sir2high subpopulations was observed: the frequency of URA3 expression was in both cases $1.4 \cdot 10^{-2}$ (Figure $2 \mathrm{~B}$ and Table S2), similar to one of the whole population with tdTomato-Sir2 (Figure 1C). We confirmed the epigenetic nature of URA3 expression by re-spreading some of these clones on 5-FOA plates, where many cells were able to grow because of repressed URA3 transcription (Figure S2). We also evaluated the timedependence of the Sir2 level by looking at the dynamics of recovery of the expression profile after cell sorting for the low- and high-expressing cells. When grown in non-selective media, the initial distribution was not restored after $6 \mathrm{~h}$ (Figure S3 and Table S2) but was restored after $24 \mathrm{~h}$ (Figure S4) in both cases. During the initial phase of recovery, the lowexpressors switched back to the initial distribution more rapidly than the high-expressors did, but still remained above the initial distribution after $6 \mathrm{~h}$. Moreover, since the high-expressors remained far below the initial distribution after $6 \mathrm{~h}$, the gap between high- and low-expressors was still large. Sir2 levels were thus relatively stable, at least in the Sir2-high subpopulation, and did not rapidly equilibrate in either subpopulation during the first $6 \mathrm{~h}$ of the initial experimental period. The initial expression profile was then recovered $24 \mathrm{~h}$ after sorting. However, considering that starving cells that are ura- in the first hours do not grow while URA+ cells start growing immediately, this difference of a few generations between initially ura- and URA+ cells should be reflected in the colony size after 3 days, the larger ones corresponding to initially URA+ cells. We therefore only scored large colonies. Switches that occurred after the first few hours were thus excluded (if new URA+ cells arose in the 6-24hr window as silencing reverts, they should have a difference of at least 3 to 12 generations compared to already URA+ cells). Nevertheless, it cannot be ruled out that the absence of a significant difference in silencing was at least partly due to the recovery of expression equilibrium during growth on SCD-ura plates. Finally, as the colony phenotypes attributed to reversion were not systematically verified on all colonies, it is not excluded that some of the small colonies can be attributed to slow growth of cells expressing URA3 at low frequency in the strain lacking Ppr1 without silencing reversion, but it should not change the interpretation of our results.

To test this hypothesis and confirm whether or not Sir2 was limiting for the silencing of telomeric URA3 in our assays, we analyzed whether changes in Sir2 levels affected silencing without using cell sorting. To this end, we increased Sir2 levels by adding a copy of SIR2 in the LEU2 locus in the UCC2210 strain. The Western-blot data highlight the twofold increase in Sir2 levels compared to the original strain (Figure $3 \mathrm{~A}$ and Table S2). Although this difference in mean expression is far from the $\sim 10$-fold difference in tdTomato-Sir2 expression in low- and high-expressing subpopulations (Figure 1A), it appears that this increased mean Sir2 expression did not affect the silencing rate (Figure 3B and Table S2), showing that changes in mean Sir2 levels have no effect on the reporter gene in the absence of cell sorting.

\section{SIR2 expression noise can generate cell-to-cell heterogeneity in viability}

Surprisingly, when measuring viability to normalize the frequency of $U R A 3$ expression, we observed a highly significant difference between the Sir2-low and Sir2-high subpopulations in our URA3 telomeric reporter strain: while 6/10 Sir2-low cells were viable, this proportion

A
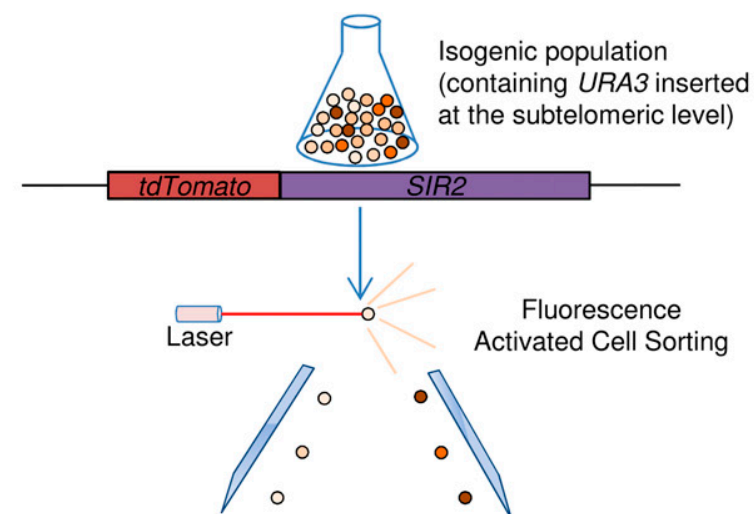

Activated Cell Sorting
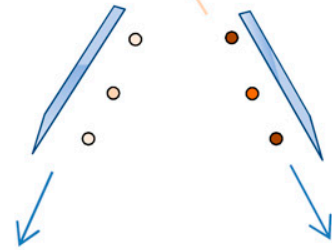

B

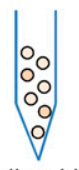

$2 \%$ cells with the lowest fluorescent levels

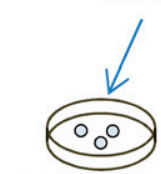

10000 cells on

URA' plate
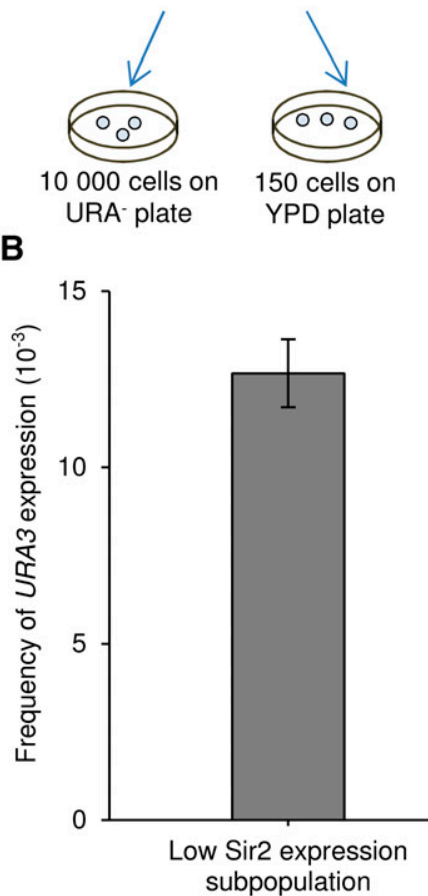

Figure 2 Noise in the expression of SIR2 does not generate cell-to-cell heterogeneity in the frequency of URA3 silencing., A) The fluorescent marker tdTomato fused $\mathrm{N}$-terminally to sir2 allows cells to be sorted by extreme expression levels (the highest $2 \%$ and the lowest 2\%). A total of $10^{4}$ cells were sorted for each subpopulation and spread on SCD-Ura plates. Viability was evaluated in parallel on YPD plates, allowing the respective frequencies of URA 3 expression in these two subpopulations to be calculated., B) Frequency of URA3 expression in the subpopulations with the highest $(2 \%)$ and lowest $(2 \%)$ cellular concentrations of tdTomato-Sir2. The results shown are the means and standard deviations of three independent experiments.

was just 2/10 among Sir2-high cells (Figure $4 \mathrm{~A}$ and Table S2) $\left(P=6.10^{-3}\right)$. The two subpopulations behave very differently: their viability is strongly influenced by their cellular concentrations of tdTomato-Sir2. 
A
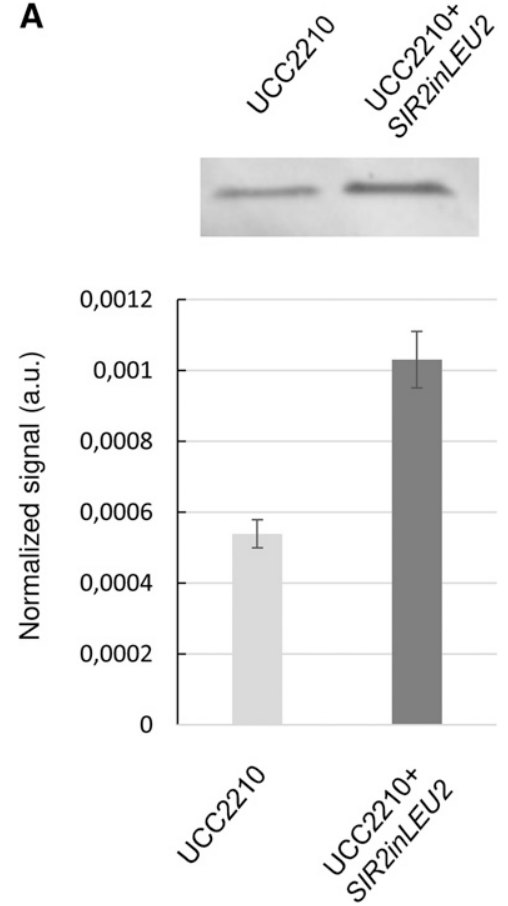

B

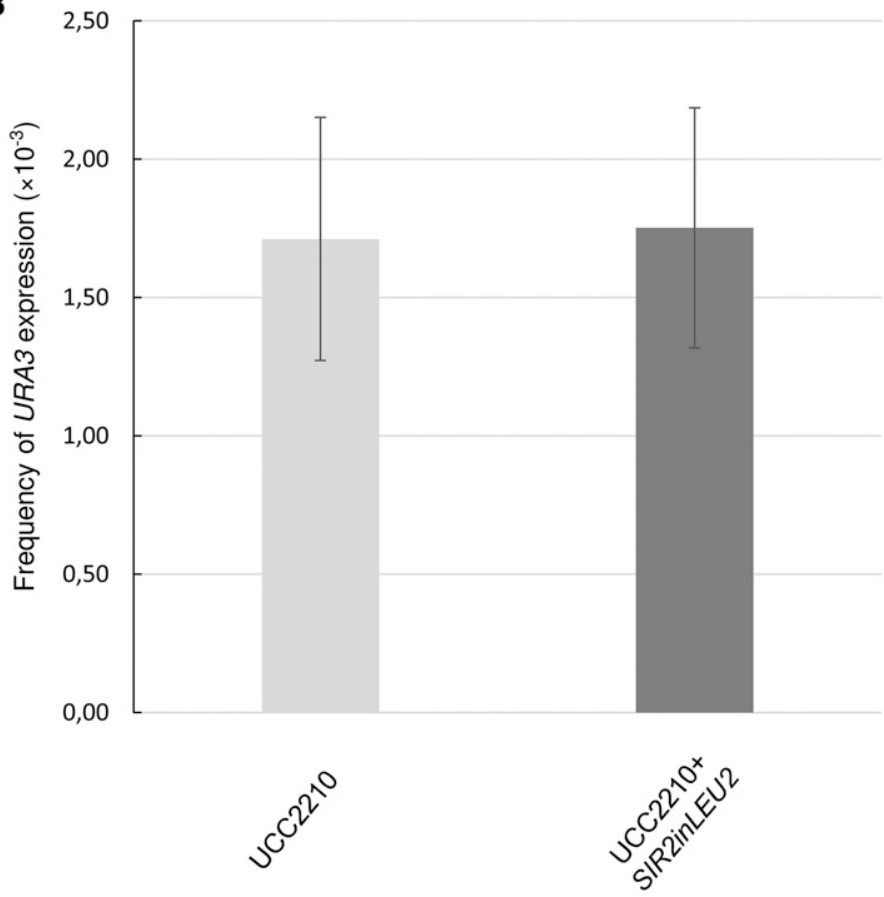

Figure 3 Doubling the mean expression level of Sir2 does not impact the frequency of URA3 silencing., A) Western blot analysis and quantification of the level of Sir2 expression in the control strain (UCC2210) and the strain with an additional copy of SIR2 inserted in the LEU2 locus (UCC2210+SIR2inLEU2) using stain-free imaging technology and the software Image Lab (Bio-Rad). The results shown are the means and mean deviations of two independent experiments (raw data of Sir2 levels and total protein levels assessment together with an example of stain free staining are provided in Table S2)., B) Frequency of URA3 expression in the control strain UCC2210 and the strain with an additional copy of SIR2 in the LEU2 locus (UCC2210+SIR2inLEU2). The results shown are the means and standard deviations of three independent experiments.

To make sure that this decrease in viability was not due to a toxic effect of tdTomato, we also sorted extreme subpopulations of a tdTomato-Tsl1 strain that contained tdTomato N-terminally fused to Tsl1 and that had the same fluorescence distribution as the strain containing tdTomato-Sir2 (Figure S5A). Contrary to the significant difference observed for Sir2 $\left(P=8.10^{-3}\right)$, no difference in viability was observed between the extreme expression levels of tdTomatoTsl1 (Figure S5B), suggesting that a toxic effect of tdTomato can be excluded. This strain was also used to verify that YPD and SCD media gave the same viability results, which is the case (Table S2).

Finally, to confirm the initial observation and to investigate whether viability scales linearly or non-linearly with Sir2 levels, we analyzed viability over the full range of Sir 2 expression by dividing the whole population into five homogenously distributed subpopulations from the lowest (subpopulation 1) to the highest (subpopulation 5) expression levels $(0-20 \%, 20-40 \%$, etc.) (Figure 4B). Viability was negatively associated with Sir2 levels (Pearson $r=-0.895$ ) (Figure 4C and Table S2). While the difference was statistically significant only between subpopulations 2 and $5(P=0.02)$, viability scaled linearly with Sir2 levels: it was similar in the $0-20 \%$ and $20-40 \%$ subpopulations and then decreased linearly from the $20-40 \%$ subpopulation to the top 20\%. These results show that Sir2 expression impacts viability not only at the highest expression levels but also at more moderate, non-extreme levels.

\section{DISCUSSION}

Gene expression is now recognized as a major source of phenotypic heterogeneity that can have profound consequences in various biological systems (Ackermann 2015). However, how epigenetics could be influenced by noise in the expression of the underlying genes has never been tested. Here, we tested the hypothesis that cell-to-cell variations in Sir2 levels could lead to differences in the frequency of epigenetic silencing in subtelomeric URA3. Our experiments allowed us to test the degree of cell-to-cell heterogeneity in epigenetic silencing due to stochastic SIR2 expression variations in the vicinity of wild-type levels. The aim was to provide more information on the sensitivity of epigenetic silencing to stochastic variations in SIR2 expression level. It appears that these fluctuations in SIR2 expression levels are not sufficient to influence silenced states of subtelomeric URA3.

Elevated levels of Sir2 have been found to not increase the silencing of a telomeric URA3 reporter gene (Renauld et al. 1993) or that of an ADE2 reporter integrated at the HMR loci (Sussel et al. 1993), but have been shown to enhance repression of RNA pol II reporters in rDNA (Smith et al. 1998), with results indicating that rDNA silencing is highly sensitive to even small changes in Sir2 levels and that there are normally limited amounts of Sir2 available for rDNA silencing (Smith et al. 1998). Therefore, one possible explanation for the fact that Sir2 expression did not alter silencing in this study is that Sir2 was not limiting for the silencing of telomeric URA3. Indeed, we found that varying Sir2 levels did not change the silencing frequency of URA3 at subtelomeres, in accordance with the literature. Nevertheless, other studies of strains carrying $A D E 2$ integrated next to the telomeric repeat of chromosome V-R have shown that Sir2 is normally limiting for maximal telomeric repression (Cockell et al. 2000), indicating a certain dependency on the reporter used. Thus, testing other genomic loci and other genes would also help to generalize our results and identify the specificity of each locus/gene pair. In any 


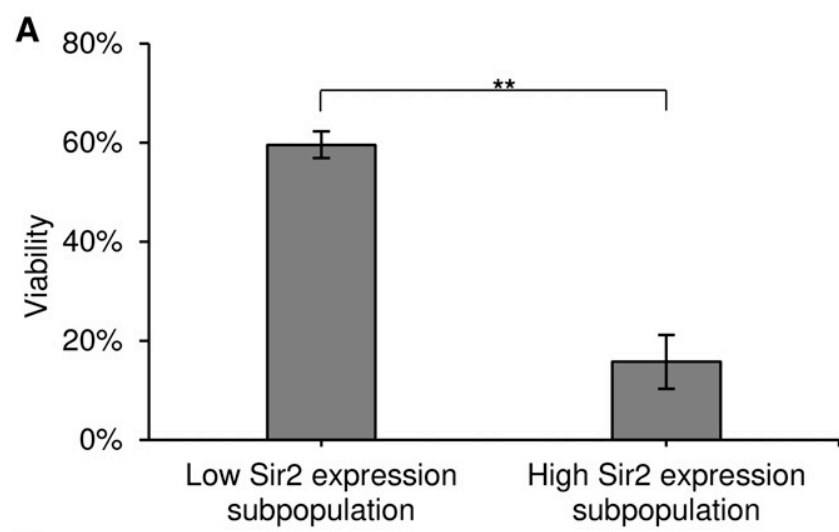

B
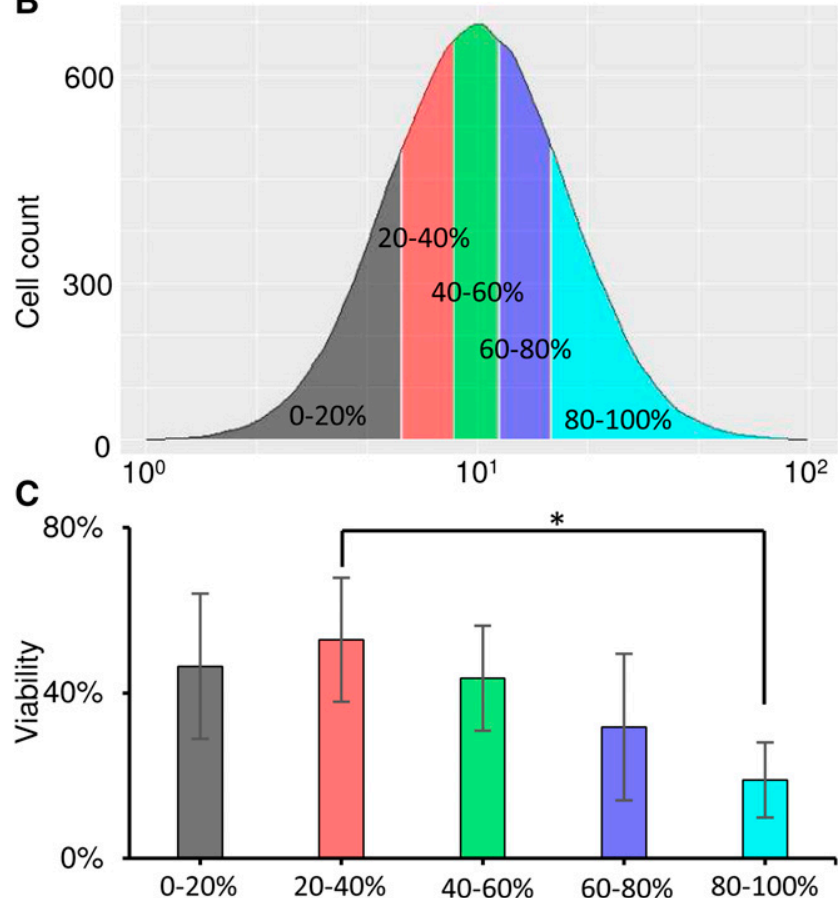

Figure 4 Noise in the expression of SIR2 can generate cell-to-cell heterogeneity in viability., A) Viability in the different subpopulations used to measure frequencies of URA3 expression in the UCC2210 strain (the $2 \%$ of cells with the lowest and highest Sir2 expression levels). The results shown are the means and standard deviations of three independent experiments. Statistically significant differences $(P<0.01, T$ tests) are indicated by double asterisks (**)., B) Five subpopulations, each representing $20 \%$ of the total population, homogenously distributed across the whole population and numbered 1 to 5 from the lowest to the highest expression levels, were sorted thanks to the fused protein tdTomato-Sir2. The same number of cells were sorted for each subpopulation and were spread on YPD plates (rich medium) to calculate viability. Three independent experiments were performed., C) Viability in these five subpopulations. The results shown are the means and standard deviations of three independent experiments. Statistically significant differences $(P<0.05$, T tests) are indicated by single asterisks $(*)$.

case, analyzing the global epigenetic landscape of the Sir2-low and Sir2high subpopulations should provide information on the short-term sensitivity of the repression of chromatin to SIR2 expression noise.

Moreover, the noise level of SIR2 may itself be minimized as observed for essential genes and protein complex subunits (Fraser et al. 2004). There are no noise measurements from systematic studies available because its expression level is too low (Newman et al. 2006). Nevertheless, it encodes for a protein complex subunit and genes with similar functions such as RPD3 whose noise has been measured indeed have low noise (Newman et al. 2006). This minimal noise would also minimize the epigenetic consequences of its expression fluctuations. Paradoxically, the targets of these chromatin remodelers are themselves expressed with high noise (Newman et al. 2006). Promoters with nucleosome binding sites generate higher cell-to-cell variability in gene expression (Sanchez and Golding 2013) while promoters with polynucleosome-disfavoring sequences have lower noise (Sharon et al. 2014). This intrinsic propensity of epigenetically regulated-genes to be noisy also suggests that noise in the expression of chromatin remodelers has to be minimized to limit downstream consequences.

Our results also show that SIR2 expression noise can generate cellto-cell heterogeneity in viability. The $2 \%$ of cells with the highest cellular concentrations of Sir 2 were far less viable than the $2 \%$ with the lowest Sir2 levels in the telomeric URA3 reporter strain, and this observation was confirmed by the general trend observed across the Sir2 expression range, with viability decreasing progressively with increasing Sir2 levels. These results, which indicate that high Sir2 subpopulations are less viable, seem contradictory to the observation that increased SIR2 gene dosage extends replicative lifespan (Kaeberlein et al. 1999; Fahrenkrog 2015). However, it has also been reported that high levels of Sir2 expression can compromise viability (Holmes et al. 1997). An explanation that can be excluded is that the decreased viability is due to the toxicity of high levels of tdTomato because a strain containing another protein N-terminally fused to tdTomato (Tsl1) that is expressed with the same fluorescence distribution as tdTomato-Sir2 does not have such heterogeneity in viability between the least and most fluorescent cells (Figure S5).

The difference in viability is surprising given that we did not observe cell-to-cell heterogeneity in silencing, suggesting that the toxic effect may be independent of transcriptional silencing. One of the many mechanisms that have been proposed to explain Sir2mediated extended lifespan (Kenyon 2010) is that it maintains gene silencing at telomeres during ageing (Dang et al. 2009). Nevertheless, high levels of Sir2 can be toxic to yeast and this effect seem rather due to decreased genome stability than to induced transcriptional silencing (Holmes et al. 1997). Note also that Sir2-induced lethality can be suppressed by H4 overexpression (Matecic et al. 2002). Thus, the viability issues caused by high Sir2 levels are almost certainly the result of a chromatin effect. In particular, if the cause is not promiscuous silencing of an essential gene, silencing of some other chromatin-based events, such as replication origins (Hoggard et al. 2018) may be involved and associated with genome instability.

While Drosophila experiments indicate that overexpression of SIR2 promotes caspase-dependent apoptosis (Griswold et al. 2008), increased Sir2 activity prevents programmed cell death caused by osmotic stress in yeast (Vendrell et al. 2011), highlighting the fact that the underlying mechanisms by which SIR2 expression or activation modulates survival remain largely unresolved. While the origins of the spontaneous cell death observed in microbial cultures even under optimal growth conditions are widely debated, multiple experimental data suggest that loss of genome integrity is a major source of apoptotic signals (Carmona-Gutierrez et al. 2010). Our work shows that noise in the expression of SIR2 is a mechanism that can induce spontaneous apoptosis in a small extreme subpopulation, and previous results suggest that possible underlying mechanisms are decreased genome stability and/or enhanced apoptotic signals. 
By examining epigenetic and phenotypic effects of cell-to-cell heterogeneity in SIR2 expression, this work shows that these fluctuations are not sufficient to produce single-cell variability in the epigenetic silencing of subtelomeric URA3 in S. cerevisiae, probably because Sir2 is not limiting for silencing subtelomeric URA3. Nevertheless, a strong effect on cell viability was observed with an almost linear relationship between decreased viability and increased Sir2 levels. The origins of this effect remain to be explored.

\section{ACKNOWLEDGMENTS}

This work was supported in part by the Agence Nationale de la Recherche (grant number ANR-12-JSV6-0006 to JPC). J.L. and J.P.C. conceived and designed the experiments. J.L., L.M. and C.B. performed experiments. J.L. and J.P.C. wrote the manuscript. J.M.F. supported the project and reviewed the manuscript. We are grateful to Delphine Lestrade and Julien Cescut from the Toulouse White Biotechnology consortium for flow cytometry facilities and to Adilia Dagkesamanskaia and Sevan Arabaciyan for their helpful contributions. We also thank Antonio Bedalov for providing the UCC2210 strain containing the URA3 silencing substrate.

\section{LITERATURE CITED}

Ackermann, M., 2015 A functional perspective on phenotypic heterogeneity in microorganisms. Nat. Rev. Microbiol. 13: 497-508. https://doi.org/ 10.1038/nrmicro3491

Anderson, M. Z., A. C. Gerstein, L. Wigen, J. A. Baller, and J. Berman, 2014 Silencing is noisy: population and cell level noise in telomereadjacent genes is dependent on telomere position and sir2. PLoS Genet. 10: e1004436. https://doi.org/10.1371/journal.pgen.1004436

Becskei, A., B. B. Kaufmann, and A. van Oudenaarden, 2005 Contributions of low molecule number and chromosomal positioning to stochastic gene expression. Nat. Genet. 37: 937-944. https://doi.org/10.1038/ ng1616

Blake, W. J., G. Balazsi, M. A. Kohanski, F. J. Isaacs, K. F. Murphy et al., 2006 Phenotypic consequences of promoter-mediated transcriptional noise. Mol. Cell 24: 853-865. https://doi.org/10.1016/j.molcel.2006.11.003

Carmona-Gutierrez, D., T. Eisenberg, S. Buttner, C. Meisinger, G. Kroemer et al., 2010 Apoptosis in yeast: triggers, pathways, subroutines. Cell Death Differ. 17: 763-773. https://doi.org/10.1038/cdd.2009.219

Cockell, M. M., S. Perrod, and S. M. Gasser, 2000 Analysis of Sir2p domains required for rDNA and telomeric silencing in Saccharomyces cerevisiae. Genetics 154: 1069-1083.

Dang, W., K. K. Steffen, R. Perry, J. A. Dorsey, F. B. Johnson et al., 2009 Histone H4 lysine 16 acetylation regulates cellular lifespan. Nature 459: 802-807. https://doi.org/10.1038/nature08085

Dey, S. S., J. E. Foley, P. Limsirichai, D. V. Schaffer, and A. P. Arkin, 2015 Orthogonal control of expression mean and variance by epigenetic features at different genomic loci. Mol. Syst. Biol. 11: 806. https://doi.org/ $10.15252 / \mathrm{msb} .20145704$

Fahrenkrog, B., 2015 Histone modifications as regulators of life and death in Saccharomyces cerevisiae. Microb. Cell 3: 1-13. https://doi.org/10.15698/ mic2016.01.472

Fraser, H. B., A. E. Hirsh, G. Giaever, J. Kumm, and M. B. Eisen, 2004 Noise minimization in eukaryotic gene expression. PLoS Biol. 2: e137. https://doi.org/10.1371/journal.pbio.0020137

Giaever, G., P. Flaherty, J. Kumm, M. Proctor, C. Nislow et al., 2004 Chemogenomic profiling: identifying the functional interactions of small molecules in yeast. Proc. Natl. Acad. Sci. USA 101: 793-798. https:// doi.org/10.1073/pnas.0307490100

Greenstein, R. A., S. K. Jones, E. C. Spivey, J. R. Rybarski, I. J. Finkelstein et al., 2018 Noncoding RNA-nucleated heterochromatin spreading is intrinsically labile and requires accessory elements for epigenetic stability. eLife 7: e32948. https://doi.org/10.7554/eLife.32948

Griswold, A. J., K. T. Chang, A. P. Runko, M. A. Knight, and K. T. Min, 2008 Sir2 mediates apoptosis through JNK-dependent pathways in
Drosophila. Proc. Natl. Acad. Sci. USA 105: 8673-8678. https://doi.org/ 10.1073/pnas.0803837105

Grunstein, M., and S. M. Gasser, 2013 Epigenetics in Saccharomyces cerevisiae. Cold Spring Harb. Perspect. Biol. 5: a017491. https://doi.org/ 10.1101/cshperspect.a017491

Hoggard, T. A., F. Chang, K. R. Perry, S. Subramanian, J. Kenworthy et al., 2018 Yeast heterochromatin regulators Sir2 and Sir3 act directly at euchromatic DNA replication origins. PLoS Genet. 14: e1007418. https:// doi.org/10.1371/journal.pgen.1007418

Holmes, S. G., A. B. Rose, K. Steuerle, E. Saez, S. Sayegh et al., 1997 Hyperactivation of the silencing proteins, Sir2p and Sir3p, causes chromosome loss. Genetics 145: 605-614.

Hornung, G., R. Bar-Ziv, D. Rosin, N. Tokuriki, D. S. Tawfik et al., 2012 Noise-mean relationship in mutated promoters. Genome Res. 22: 2409-2417. https://doi.org/10.1101/gr.139378.112

Kaeberlein, M., M. McVey, and L. Guarente, 1999 The SIR2/3/4 complex and SIR2 alone promote longevity in Saccharomyces cerevisiae by two different mechanisms. Genes Dev. 13: 2570-2580. https://doi.org/10.1101/ gad.13.19.2570

Kenyon, C. J., 2010 The genetics of ageing. Nature 464: 504-512. https:// doi.org/10.1038/nature08980

Liu, J., J. M. Francois, and J. P. Capp, 2019 Gene Expression Noise Produces Cell-to-Cell Heterogeneity in Eukaryotic Homologous Recombination Rate. Front. Genet. 10: 475. https://doi.org/10.3389/fgene.2019.00475

Liu, J., H. Martin-Yken, F. Bigey, S. Dequin, J. M. Francois et al., 2015 Natural yeast promoter variants reveal epistasis in the generation of transcriptional-mediated noise and its potential benefit in stressful conditions. Genome Biol. Evol. 7: 969-984. https://doi.org/10.1093/gbe/ evv047

Lum, P. Y., C. D. Armour, S. B. Stepaniants, G. Cavet, M. K. Wolf et al., 2004 Discovering modes of action for therapeutic compounds using a genome-wide screen of yeast heterozygotes. Cell 116: 121-137. https:// doi.org/10.1016/S0092-8674(03)01035-3

Mano, Y., T. J. Kobayashi, J. Nakayama, H. Uchida, and M. Oki, 2013 Single cell visualization of yeast gene expression shows correlation of epigenetic switching between multiple heterochromatic regions through multiple generations. PLoS Biol. 11: e1001601. https://doi.org/10.1371/ journal.pbio.1001601

Matecic, M., S. Stuart, and S. G. Holmes, 2002 SIR2-induced inviability is suppressed by histone H4 overexpression. Genetics 162: 973-976.

Newcomb, B., and A. Bedalov, 2009 Identification of inhibitors of chromatin modifying enzymes using the yeast phenotypic screens. Methods Mol. Biol. 548: 145-160. https://doi.org/10.1007/978-1-59745-540-4_8

Newman, J. R., S. Ghaemmaghami, J. Ihmels, D. K. Breslow, M. Noble et al., 2006 Single-cell proteomic analysis of S. cerevisiae reveals the architecture of biological noise. Nature 441: 840-846. https://doi.org/10.1038/ nature 04785

Obersriebnig, M. J., E. M. Pallesen, K. Sneppen, A. Trusina, and G. Thon, 2016 Nucleation and spreading of a heterochromatic domain in fission yeast. Nat. Commun. 7: 11518. https://doi.org/10.1038/ncomms11518

Raj, A., and A. van Oudenaarden, 2008 Nature, nurture, or chance: stochastic gene expression and its consequences. Cell 135: 216-226. https:// doi.org/10.1016/j.cell.2008.09.050

Rawal, Y., R. V. Chereji, H. Qiu, S. Ananthakrishnan, C. K. Govind et al., 2018 SWI/SNF and RSC cooperate to reposition and evict promoter nucleosomes at highly expressed genes in yeast. Genes Dev. 32: 695-710. https://doi.org/10.1101/gad.312850.118

Renauld, H., O. M. Aparicio, P. D. Zierath, B. L. Billington, S. K. Chhablani et al., 1993 Silent domains are assembled continuously from the telomere and are defined by promoter distance and strength, and by SIR3 dosage. Genes Dev. 7: 1133-1145. https://doi.org/10.1101/gad.7.7a.1133

Sanchez, A., S. Choubey, and J. Kondev, 2013 Regulation of noise in gene expression. Annu. Rev. Biophys. 42: 469-491. https://doi.org/10.1146/ annurev-biophys-083012-130401

Sanchez, A., and I. Golding, 2013 Genetic determinants and cellular constraints in noisy gene expression. Science 342: 1188-1193. https://doi.org/ $10.1126 /$ science. 1242975 
Sharon, E., D. van Dijk, Y. Kalma, L. Keren, O. Manor et al., 2014 Probing the effect of promoters on noise in gene expression using thousands of designed sequences. Genome Res. 24: 1698-1706. https://doi.org/10.1101/ gr.168773.113

Simon, J. A., and A. Bedalov, 2004 Yeast as a model system for anticancer drug discovery. Nat. Rev. Cancer 4: 481-492. https://doi.org/10.1038/nrc1372

Small, E. C., L. Xi, J. P. Wang, J. Widom, and J. D. Licht, 2014 Single-cell nucleosome mapping reveals the molecular basis of gene expression heterogeneity. Proc. Natl. Acad. Sci. USA 111: E2462-E2471. https:// doi.org/10.1073/pnas.1400517111

Smith, J. S., C. B. Brachmann, L. Pillus, and J. D. Boeke, 1998 Distribution of a limited Sir2 protein pool regulates the strength of yeast rDNA silencing and is modulated by Sir4p. Genetics 149: 1205-1219.

Sussel, L., D. Vannier, and D. Shore, 1993 Epigenetic switching of transcriptional states: cis- and trans-acting factors affecting establishment of silencing at the HMR locus in Saccharomyces cerevisiae. Mol. Cell. Biol. 13: 3919-3928. https://doi.org/10.1128/MCB.13.7.3919

To, T. L., and N. Maheshri, 2010 Noise can induce bimodality in positive transcriptional feedback loops without bistability. Science 327: 1142-1145. https://doi.org/10.1126/science.1178962

Uphoff, S., N. D. Lord, B. Okumus, L. Potvin-Trottier, D. J. Sherratt et al., 2016 Stochastic activation of a DNA damage response causes cell-to-cell mutation rate variation. Science 351: 1094-1097. https://doi.org/10.1126/ science.aac9786

Vendrell, A., M. Martinez-Pastor, A. Gonzalez-Novo, A. Pascual-Ahuir, D. A. Sinclair et al., 2011 Sir2 histone deacetylase prevents programmed cell death caused by sustained activation of the Hog1 stress-activated protein kinase. EMBO Rep. 12: 1062-1068. https://doi.org/10.1038/ embor.2011.154

Viñuelas, J., G. Kaneko, A. Coulon, E. Vallin, V. Morin et al., 2013 Quantifying the contribution of chromatin dynamics to stochastic gene expression reveals long, locus-dependent periods between transcriptional bursts. BMC Biol. 11: 15. https://doi.org/10.1186/1741-700711-15

Weinberger, L., Y. Voichek, I. Tirosh, G. Hornung, I. Amit et al., 2012 Expression noise and acetylation profiles distinguish HDAC functions. Mol. Cell 47: 193-202. https://doi.org/10.1016/ j.molcel.2012.05.008

Xu, E. Y., K. A. Zawadzki, and J. R. Broach, 2006 Single-cell observations reveal intermediate transcriptional silencing states. Mol. Cell 23: 219-229. https://doi.org/10.1016/j.molcel.2006.05.035

Zaugg, J. B., and N. M. Luscombe, 2012 A genomic model of conditionspecific nucleosome behavior explains transcriptional activity in yeast. Genome Res. 22: 84-94. https://doi.org/10.1101/gr.124099.111

Zhang, Z., W. Qian, and J. Zhang, 2009 Positive selection for elevated gene expression noise in yeast. Mol. Syst. Biol. 5: 299. https://doi.org/10.1038/ msb. 2009.58

Zoller, B., D. Nicolas, N. Molina, and F. Naef, 2015 Structure of silent transcription intervals and noise characteristics of mammalian genes. Mol. Syst. Biol. 11: 823. https://doi.org/10.15252/msb.20156257

Communicating editor: J Berman 